\title{
Variability of Brain Signals Processed Locally Transforms into Higher Connectivity with Brain Development
}

\author{
Vasily A. Vakorin, ${ }^{1}$ Sarah Lippé, ${ }^{2}$ and Anthony R. McIntosh ${ }^{1,3}$ \\ ${ }^{1}$ Rotman Research Institute of Baycrest, Toronto, Ontario M6A 2E1, Canada, ${ }^{2}$ Sainte-Justine Hospital Research Centre, Department of Psychology, \\ University of Montreal, Montreal, Quebec H3T 1C5, Canada, and '3Department of Psychology, University of Toronto, Toronto, Ontario M5S 3G3, Canada
}

\begin{abstract}
A number of studies have characterized the changes in variability of brain signals with brain maturation from the perspective of considering the human brain as a complex system. Specifically, it has been shown that complexity of brain signals increases in development. On one hand, such an increase in complexity can be attributed to more specialized and differentiated brain regions able to express a higher repertoire of mental microstates. On the other hand, it can be explained by increased integration between widely distributed neuronal populations and establishment of new connections. The goal of this study was to see which of these two mechanisms is dominant, accounting for the previously observed increase in signal complexity. Using information-theoretic tools based on scalp-recorded EEG measurements, we examined the trade-off between local and distributed variability of brain signals in infants and children separated into age groups of 1-2,2-8,9-24, and 24-66 months old. We found that developmental changes were characterized by a decrease in the amount of information processed locally, with a peak in alpha frequency range. This effect was accompanied by an increase in the variability of brain signals processed as a distributed network. Complementary analysis of phase locking revealed an age-related pattern of increased synchronization in the lower part of the spectrum, up to the alpha rhythms. At the same time, we observed the desynchronization effects associated with brain development in the higher beta to lower gamma range.
\end{abstract}

\section{Introduction}

Many theoretical aspects of brain function focus on a controversy between localized and distributed properties of the structure and dynamics of the large-scale neuronal networks supporting a specific cognitive or behavioral task (Cohen and Tong, 2001). Under this account, two complementary principles are established in the literature: functional segregation and integration (Friston, 1998). Evidence in support of functionally segregated organization at different levels of the hierarchy is extensive. In contrast to modular deployment of local specialization, neuronal groups are interconnected between each other at many levels to support the emergence of coherent cognitive and behavioral states (Churchland and Sejnowski, 1988; Jirsa and McIntosh, 2007).

A number of studies have characterized changes in variability of brain signals with brain maturation (e.g., McIntosh et al., 2008; Lippe et al., 2009). These studies focused on using the concept of complexity, a broadly defined property characterizing a highly variable system with many parts whose behaviors strongly depend on the behavior of other parts (Deisboeck and Kresh, 2006). Meyer-Lindenberg (1996) analyzed the changes in resting electroencephalography (EEG) measurements from children during normal brain development. McIntosh et al. (2008) measured the complexity of EEG signals from children and young adults per-

\footnotetext{
Received June 17, 2010; revised Jan. 19, 2011; accepted Jan. 25, 2011.

This research was supported by grants from the J.S. McDonnell Foundation.

We thank Maria Tassopoulos for her assistance in preparing this manuscript.

Correspondence should be addressed to Vasily A. Vakorin at the above address. E-mail: vasenka@gmail.com.

DOI:10.1523/JNEUROSCI.3153-10.2011

Copyright $\odot 2011$ the authors $\quad 0270-6474 / 11 / 316405-09 \$ 15.00 / 0$
}

forming a face memory task, accounting for the multiple timescale inherent in the electrode measurements with the multiscale entropy (Costa et al., 2002). They found that brain signal variability increased with age, negatively correlating with variability in reaction time and positively correlating with accuracy. Similar results were reported by Lippé et al. (2009), who showed that EEG signal complexity increased from 1 month old to 5 years in response to auditory and visual stimuli.

Certain fundamental properties of anatomical and functional organization of a network can affect the interplay between functional segregation and integration. This may include either locally processed information or redirecting information flows to an assembly of neuronal populations distributed across many different regions of the brain. In other words, there are basically two mechanisms available to respond to an increased demand for computational load. On one hand, the observed increase in complexity of the brain signals can be attributed to more specialized and differentiated individual brain regions, which are able to demonstrate a bigger repertoire of neural dynamics. On the other hand, a higher level of integration between neuronal populations and establishment of new connections can account for the increased brain complexity in development (Tononi et al., 1994).

This paper studied the interplay between segregation and integration of information in brain development in terms of functional variability of neurosignals. Methodologically, this study was an attempt to decompose the total variability of the signals associated with specific neuronal populations into local entropy, attributed to their individual dynamics, and distributed entropy, which characterizes their behavior affiliated with dynamics of 
other populations. The specific objective was to test which of these two mechanisms, if either, was the dominant factor contributing to an increased complexity of brain signals associated with brain development. Data-driven information-theoretic tools were applied to estimate mutual information between interacting brain areas and remaining uncertainty of individual modules of the network. As the EEG patterns in the study may reflect not only induced responses, but also evoked components, we also analyzed the changes in phase locking based on the concept of intertrial coherence (Tass et al., 1998).

\section{Materials and Methods}

EEG data acquisition. Forty infants and children ( $n=40,13$ females, 1 of gender unregistered) of age $27 \mathrm{~d}$ to 5 years 5 months were involved in this study. Data from five participants were excluded from the analysis because of excessive movements artifacts or failure in attending to both the visual and auditory stimuli. Infants and children were separated into four age groups: $1-2$ months ( $n=7,3$ females), $2-8$ months $(n=11,4$ females), $9-24$ months ( $n=6,3$ females), and $24-66$ months ( $n=11,3$ females, 1 of gender unregistered). The data we analyzed were previously used by Lippé et al. $(2007,2009)$.

EEG data were acquired using the 128-channel Electrical Geodesics Incorporated system. Impedances were kept below $40 \mathrm{k} \Omega$. EEG data were digitized at $250 \mathrm{~Hz}$ sampling rate and passed through a $0.01-100 \mathrm{~Hz}$ bandpass filter. During the recording, all electrodes were referenced to the vertex but were re-referenced to an average reference for further analysis. More details on the acquisition protocol have been given by Lippé et al. (2007).

The study applied two type of stimuli: auditory and visual. The auditory stimulus was a $50 \mathrm{~ms}$ broadband noise with the intensity of $70 \mathrm{~dB}$, presented in free-field binaurally in a soundproof room. The noise was delivered with an Optimus a XTS 24 system located at a distance of $30 \mathrm{~cm}$ from each of the subject's ears. The visual stimulus was a black-and-white checkerboard presented binocularly for $500 \mathrm{~ms}$ at a distance of $70 \mathrm{~cm}$ from the subject's eyes with a luminance of $40 \mathrm{~cd} / \mathrm{m}^{2}$. Each square of the checkerboards delimited a visual angle of $2 \times 2^{\circ}$, whereas the checkerboards themselves subtended $38 \times 38^{\circ}$ of visual angle. The E-Prime 2000 software (Psychology Software Tools) was used to generate all the stimuli.

Local and distributed entropy. Information theory provides important tools and a unified framework for studying complex systems. Such methods found wide applicability in neuroscience (Baddeley et al., 2000). Entropy and mutual information are two basic concepts of the information theory (Shannon, 1949). Entropy $H(X)$ is a measure of uncertainty associated with a single random variable $X$. It is possible to define conditional entropy $H(X \mid Y)$, which is the entropy of a random variable, provided that the knowledge about another random variable is excluded. The reduction in uncertainty due to another variable is called mutual information. Specifically, the mutual information between two random variables is as follows:

$$
I(X ; Y)=H(X)-H(X \mid Y) .
$$

The mutual information is a measure of affiliation between two variables. By construction, it is non-negative. Zero values of $I(X ; Y)$ mean independence between $X$ and $Y$. The higher the values of $I(X ; Y)$, the higher the degree of affiliation between $X$ and $Y$.

In addition, mutual information is a symmetric measure. Specifically,

$$
I(X ; Y)=I(Y ; X)=H(Y)-H(Y \mid X),
$$

where $H(Y)$ is the entropy of the variable $Y$, and $H(Y \mid X)$ is the conditional entropy of $Y$, given the uncertainty contained in $X$.

Note that the mutual information of a random variable with itself is equal to the entropy of this variable:

$$
I(X ; X)=H(X)-H(X \mid X)=H(X),
$$

which gives the idea why the entropy might be referred to as expected self-information.
The joint entropy $H(X, Y)$, which represents the uncertainty of a pair of random variables $(X, Y)$, can be partitioned into the conditional (pure) entropies of the variables $X$ and $Y$, and the mutual information between them. Specifically,

$$
H(X, Y)=H(X \mid Y)+H(Y \mid X)+I(X ; Y) .
$$

Suppose that a network is identified and consists of $M$ nodes (electrodes in our case), interacting with each other. Next, consider a pair of nodes, $i$ and $j$, represented by two variables, $X_{i}$ and $X_{j}$, where $i \in[1, M], j \in[1$, $M]$, and $i \neq j$. In the context of pairwise connections, the information contained in a node $i$ can be partitioned into the local entropy associated only with the complexity of the node $i$ and the distributed entropy that is shared between $i$ and $j$. The local entropy corresponds to the conditional entropy $H\left(X_{i} \mid X_{j}\right)$, whereas the distributed entropy $E_{\text {distrib }}(i, j)$ is represented by the mutual information:

$$
E_{\text {distrib }}(i, j)=I\left(X_{i} ; X_{j}\right)
$$

In turn, local entropy $E_{\text {local }}(i)$ is associated with conditional entropy. In this study, for a given node $i$, the local entropy will be averaged over the rest of the nodes from the network. Specifically,

$$
E_{\text {local }}(i)=\frac{1}{M-1} \sum_{\substack{j=1 \\ j \neq i}}^{M} H\left(X_{i} \mid X_{j}\right) .
$$

Entropy estimation. Until now, we described the theoretical aspects of partitioning the total entropy of a given signal into the two components, local and distributed. Still, estimating entropy based on finite noisy time series may be of crucial importance. Methods developed under the framework of nonlinear dynamical systems may give more accurate estimates of the entropies than the standard approaches such as boxcounting techniques (Prichard and Theiler, 1995).

Specifically, entropies can be quantified in terms of nonlinear dynamics. The rationale for such an approach is that many complex biophysiological phenomena are due to nonlinear effects. Recently there has been an increasing interest in studying complex neural networks in the brain, applying concepts and time series analysis techniques derived from nonlinear dynamics (Stam, 2005). Under this context, we assume that the dynamics of the underlying multidimensional system can be reconstructed from the observed time series. This can be achieved by a technique called time delay embedding, in which the underlying process is represented by the same versions of the original signal shifted in time. The underlying dynamics is associated with a sequence of points in a multidimensional space with the coordinates obtained by taking the amplitude values of the original time series at several consecutive times, separated by a time lag (usually measured in data points).

Let $x_{t}^{(i)}$ be realizations of the variable $X_{i}$ observed as measurements from the $i$ th electrode, where $i=1, \ldots, M$. Then, $x_{t}^{(i)}$ denotes the delay vector at the time $t$, describing the recent history of the process $X_{i}$ as follows:

$$
x_{t}^{(i)}=\left(x_{t}^{(i)}, x_{t-\tau_{i}}^{(i)}, \ldots, x_{t-\tau_{i}\left(d_{i}-1\right)}^{(i)}\right)^{T},
$$

where $d_{i}$ is the embedding dimension, and $\tau_{i}$ is the embedding delay measured in multiples of the sampling interval. The sign $\mathrm{T}$ stands for transpose.

In general, time delay embedding is a crucial and nontrivial step. There are many competing approaches proposed in the literature, and all of them are heuristic and somewhat mutually exclusive. A number of studies have proposed different information-theoretic criteria for selecting the embedding parameters optimally. In this study, we followed Small and Tse (2004), who proposed a robust criterion for reconstructing the underlying dynamics from a finite time series in the presence of noise. Their conclusion was that only the embedding window $d_{i} \times \tau_{i}$ is significant, whereas the lag $\tau_{i}$ is model dependent. Their method proved to be robust and consistent, and worked for relatively short or noisy time series. In our study, most of the time series for all the participants in both conditions, with a few exceptions, were characterized by the embedding 

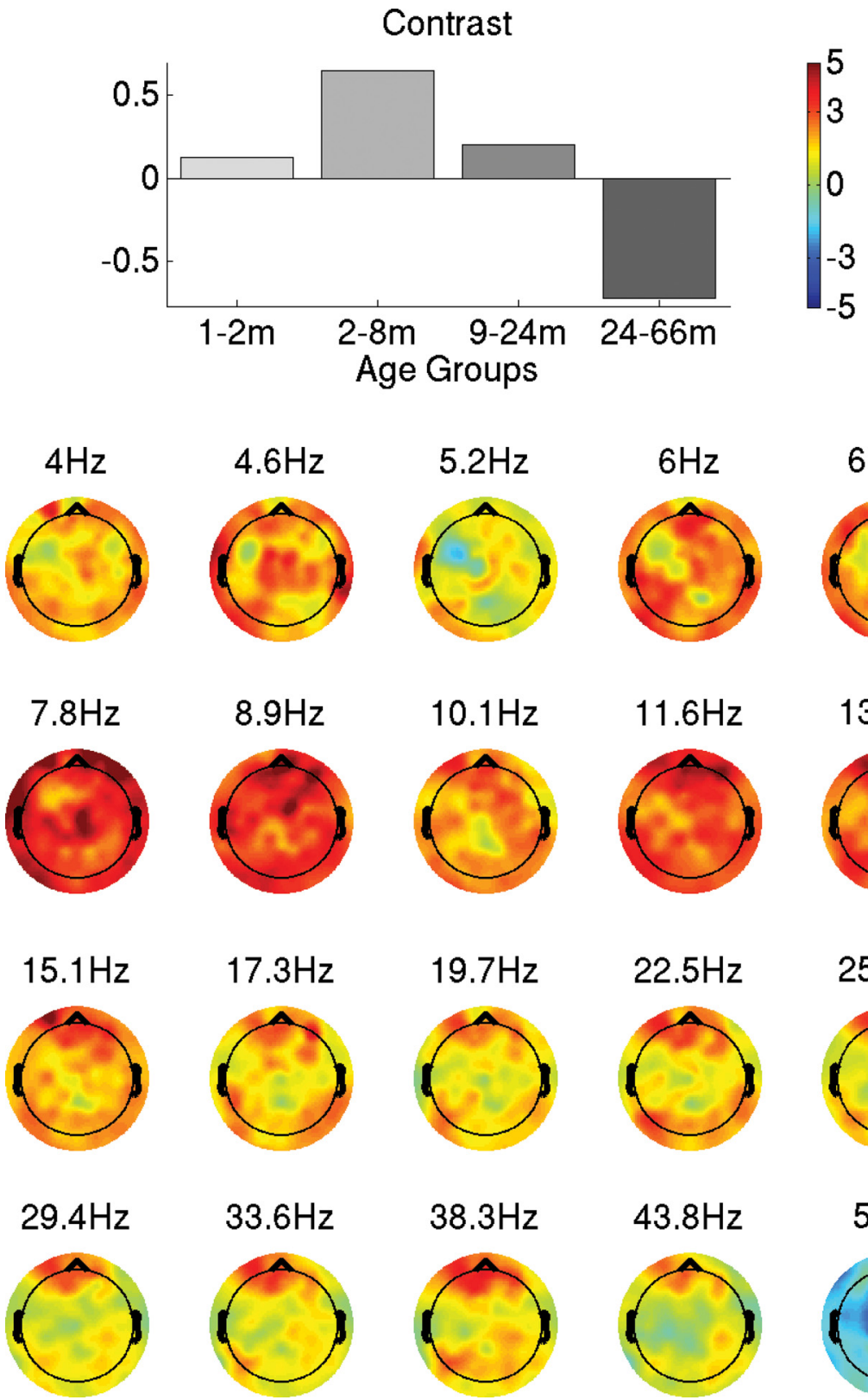

Figure 1. Local entropy: age-related changes in the variability of signals associated with local dynamics in auditory condition. The pattern of changes represented by the contrast from PLS analysis is shown in the upper part. The topographic (PLSbootstrap ratio) maps reflect the spatial distribution of electrode loadings, showing the electrodes' contribution at a specific frequency to the identified contrast.

window estimated to equal 2, implying the embedding dimension $d_{i}=2$ and the embedding delay $\tau_{i}=1$.

We use the reconstructed dynamics $\boldsymbol{x}^{(i)}$ to estimate the individual, joint, and conditional entropies as used in Equation 4. The original approach is to divide the state space into $K$ bins of some size $\delta$ and calculate the entropy $H(\boldsymbol{x})$ of multidimensional dynamics $\boldsymbol{x}=\left\{\boldsymbol{x}_{t}\right\}_{t-1}^{N}$, observed $N$ times at time $t$, through constructing a histogram, estimating probabilities $p_{k}(\boldsymbol{x}, \delta)$ of being in the $k$ th bin. The signal $\boldsymbol{x}$ is treated as realizations of a discrete random variable, and the entropy is defined by

$$
H(\boldsymbol{x}, \delta)=-\sum_{k=1}^{K} p_{k}(\boldsymbol{x}, \delta) \log _{2} p_{k}(\boldsymbol{x}, \delta) .
$$

Another approach is to estimate the corresponding correlation integrals, which may give more accurate estimates than box-counting techniques
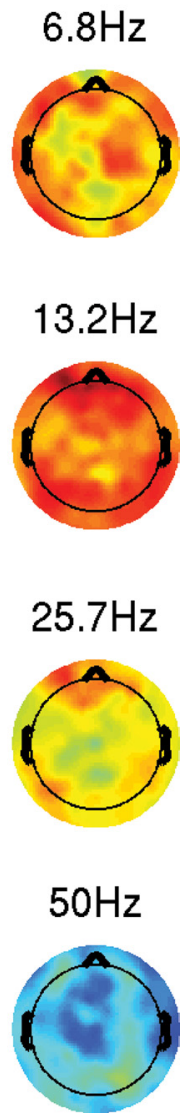

(Prichard and Theiler, 1995). Specifically, entropy is related to the correlation integral $C_{q}(\boldsymbol{x}, r)$ through

$$
\begin{array}{r}
H(\boldsymbol{x}, \delta)=-\sum_{k(\text { bins })} p_{k}(\boldsymbol{x}, \delta) \log _{2} p_{k}(\boldsymbol{x}, \delta) \approx \\
-\log _{2} C_{q}(\boldsymbol{x}, r)
\end{array}
$$

as a function of the characteristic scale length $r$, and the integral order $q$ (Prichard and Theiler, 1995). The parameter $r$ can be related to the bin size $\delta$.

The correlation integral is approximated by the correlation sum $C_{q}(\boldsymbol{x}, r)$ estimated as follows:

$$
\begin{array}{r}
C_{q}(\boldsymbol{x}, r)=\frac{1}{N(N-1)^{q-1}} \times \sum_{s=1}^{N}\left[\sum_{t \neq s} \Theta(r-\right. \\
\left.\left.\left\|\boldsymbol{x}_{s}-\boldsymbol{x}_{t}\right\|\right)\right],
\end{array}
$$

where $N$ is the number of data points, $\Theta$ is the Heaviside function, and $\|\cdot\|$ stands for the maximum norm distance between two data points $\boldsymbol{x}_{s}$ and $\boldsymbol{x}_{t}$. The second-order $(q=2)$ correlation integral is interpreted as the likelihood that the distance between two randomly chosen points, $\boldsymbol{x}_{s}$ and $\boldsymbol{x}_{t}$ in the multidimensional space spanned by $\boldsymbol{x}$, at times $s$ and $t$, is smaller than $r$. The function $\Sigma_{t \neq s} \Theta\left(r-\left\|\boldsymbol{x}_{s}-\boldsymbol{x}_{t}\right\|\right)$ for a given point $s$ represents the number of points $t$ such that the distance between the $d$-dimensional vectors $\boldsymbol{x}_{s}$ and $\boldsymbol{x}_{t}$ is less than $r$. The expression $(1 / N) \Sigma_{s \neq t} \Theta\left(r-\left\|\boldsymbol{x}_{s}-\boldsymbol{x}_{t}\right\|\right)$ in Equation 10 is a specific case of kernel density estimation, which is generally considered superior to calculating entropy through estimating a multidimensional histogram (Silverman, 1986).

Phase locking. The analysis of brain connectivity in terms of signal amplitudes was complemented by computing a measure called phase-locking index (PLI) (Chávez et al., 2003; Stam et al., 2007). The PLI is known in the literature under different names, such as mean phase coherence (Mormann et al., 2000) or phase synchronization index (Tass et al., 1998). The PLI is able to quantify phase synchronization between signals in a statistical sense, and emerged from studying coupled nonlinear systems (Rosenblum et al., 1996). In turn, phase synchronization is based on an idea that the existence of relations between phases of coupled systems does not necessarily imply correlation between their amplitudes.

Specifically, phase locking between channels across trials was computed using the concept of frequency-specific phase difference between the signals. More specifically, the cross spectrum $S_{i j}$ as a function of frequency $f$ between two signals $X_{i}$ and $Y_{j}$ has

$$
S_{i j} \sim \exp \left(i \phi_{i j}(f)\right),
$$

where $\phi_{i j}(f)$ is the cross-phase spectrum. The function $\phi_{i, j}(f)$ represents the phase delay between the two signals at a specific frequency $f$.

Suppose that there are $K$ estimates (for each trial) of the cross-phase spectra. The functions $\exp \left(i \phi_{i j}(f)\right), k=1, \ldots, K$ represent the radius vectors in the complex space. The phase-locking index $R_{i j}(f)$ is computed as the absolute value of the mean vector obtained by averaging the radius 
vectors associated with cross-phase spectra, across trials in the complex space. Specifically,

$$
R_{i j}(f)=\left|\frac{1}{K} \sum_{k=1}^{K} \exp \left(i \phi_{i j}(f)\right)\right| .
$$

The phase-locking index represents a measure to quantify the amount of phase synchrony inherent in two given signals across repeated exposure to stimuli. By design, the statistic $R_{i j}(f)$ is limited between 0 and 1 . When the crossphase distribution is concentrated around the mean, the PLI is close to 1 . The PLI is close to 0 for the uniformly distributed phase differences across trials.

Partial least squares. Partial least-squares (PLS) analysis is a multivariate technique based on an idea of extracting latent factors that account for most of the variance of data under investigation. This study takes the mean-centering approach wherein the meancentered matrix is decomposed to produce a set of mutually orthogonal factors. Here we give a brief description of the technique, and refer the reader to Lobaugh et al. (2001), Hay et al. (2002), Düzel et al. (2003), and McIntosh and Lobaugh (2004) for more details.

PLS operates on the entire data structure at once with the data organized in a matrix form. The rows of the data correspond to subjects within conditions, whereas the columns correspond to elements such as voxels in functional magnetic resonance imaging, electrodes in EEG, or magnetoencephalography sensors. In our case, the elements are represented by individual electrodes for the analysis based on local entropy, and all the possible pairs of electrodes for mutual information and phase locking. Then, two steps are performed. First, columnwise statistic averages are computed within each condition. Second, the original data matrix is mean-centered with respect to the condition-specific statistic average of the entire column.

Next, singular value decomposition (SVD) is used to project the data matrix to a set of orthogonal latent variables (LVs), which are the left and right singular vectors from the SVD with decreasing order of magnitude (analogously to principal component analysis). Thus, a latent variable consists of three components. It includes the following: (1) a singular value; (2) a vector of the condition loadings (weights within the right singular vector) that represent an underlying contrast; and (3) a vector of the element loadings (weights within the left singular vector) that represent the optimal relation of the elements to the identified contrast.

Statistical assessment regarding the number of LVs to retain and the importance of individual element weights within a specific LV is based on resampling procedures. The first step is performed using permutation tests, which randomly reassign conditions within subjects. The permutation test assesses the significance of the effect represented in a given $\mathrm{LV}$, in a sense how it is different from random noise. A measure of significance is calculated as the number of times the permuted singular value is higher than the observed singular value. In the second step, the element loadings are further tested for stability across subjects through bootstrap resampling of subjects within conditions. A measure of stability is calculated as the ratio of the loading to the SE of the generated bootstrap distribution, and is approximately equivalent to a $z$-score. For example, the absolute bootstrap ratios $>3.5$ correspond approximately to a $99 \%$ confidence interval. Furthermore, elements with positive bootstrap ratio values directly support the contrast associated with a given LV. Elements with negative bootstrap ratio values may also support the underlying contrast, but in a reverse way.

Pipeline of the analysis. The first step of the analysis was performed with EEGLAB (Delorme and Makeig, 2004). The data from 18 electrodes were discarded due to the presence of excessive artifacts, thus leaving 110 electrodes for the further analysis. The continuous EEG recordings were segmented into the auditory and visual stimuli events on the [-100 488] ms intervals. In addition, independent component analysis (ICA) was performed for artifact removal and correction as follows. First, we removed trials contaminated with excessive amplitudes. Then, the subjectbased remaining concatenated trials were decomposed with ICA. Each independent component was examined in terms of its topography, power spectrum, activity over time and trials. Components associated with residual ocular and muscle artifacts were identified based on topography and frequency, and were removed from the dataset. Sixty good 
trials were kept for each subject and condition. On preprocessing, see more details in the Lippé et al. (2009) report.

The phase-locking analysis was performed as specified above, in Phase locking, for each pair of electrodes. The entropy analysis was performed based on spectrally decomposed data. Specifically, the time-frequency representation of the original time series was derived by convolving the data with the Morlet wavelets. Twenty frequency points were logarithmically equally spaced between 4 and $50 \mathrm{~Hz}$. The following parameters were used: the embedding dimension $d=2$, the embedding lag $\tau=1$, and the characteristic scale length $r=1$. The distributed and local entropy as defined in Equations 5 and 6, respectively, were estimated for all the single trials. Then, the entropy statistics defined in Equations 5 and 6 were averaged over trials within conditions.

To prepare for PLS analysis, the data matrices were organized in the form of (age groups $X$ subjects) by elements (electrodes or pairs of electrodes). Six mean-centered PLS analyses were performed to explore changes in phase locking, distributed and local entropy in auditory and visual conditions, separately. The significance of LV's contrast was estimated using permutation tests with 500 permutations. The contribution of individual electrodes (for the local entropy analyses) and connections between the electrodes (for the distributed entropy and phase-locking analyses) to the identified contrasts was assessed using bootstrap resampling with 500 samples.

\section{Results}

The following sections describe how three qualities, namely local and distributed entropy and the degree of phase locking in terms of intertrial coherence, change across four aging groups as the functions of frequency.

\section{Local entropy}

The PLS analyses revealed the existence of one significant LV for each modality $(p<0.001)$, which can be interpreted as the standpoint of developmental changes. Specifically, Figures 1 and 2 show the age-related patterns of changes in the local entropy statistic $E_{\text {local }}$ in the auditory and visual conditions, respectively. The upper parts of the figures, which represent the corresponding data-driven contrasts, illustrate the developmental trends in the variability of brain signals, associated with local processing.

With the exception of the increase in the amount of local entropy for babies of 2-8 months old compared to 1-2 months in the auditory task, both auditory (Fig. 1, gray contrast) and visual (Fig. 2, copper contrast) conditions are in general characterized by a decrease in local entropy with maturation of the brain. It should be noted, however, that the contrast in PLS is a relative measure. The contrast should be interpreted only considering the question of how exactly it is expressed across the elements (electrodes and frequencies in our case).

Electrodes' contribution is shown as the topographic maps of the bootstrap ratio values, reflecting the extent to which individual electrodes at specific frequencies support the identified contrast. Positive bootstrap ratio values coded in red directly support the contrast under investigation. Negative bootstrap ratio values shown in blue indicate electrodes for which the contrast should be inverted (multiplied by -1 ). Electrodes of robust contrast expression can be identified by thresholding the absolute bootstrap ratios to values $>3.5$, corresponding approximately to a 99\% confidence interval. With regards to the auditory task (Fig. $1)$, the positive peaks on the topographic maps are observed at 8-9 Hz. Similar peaks can be found for the visual task (Fig. 2) around $8 \mathrm{~Hz}$.

\section{Distributed entropy}

A decrease in the variability of signals related to local processing is accompanied by the expected complementary developmental
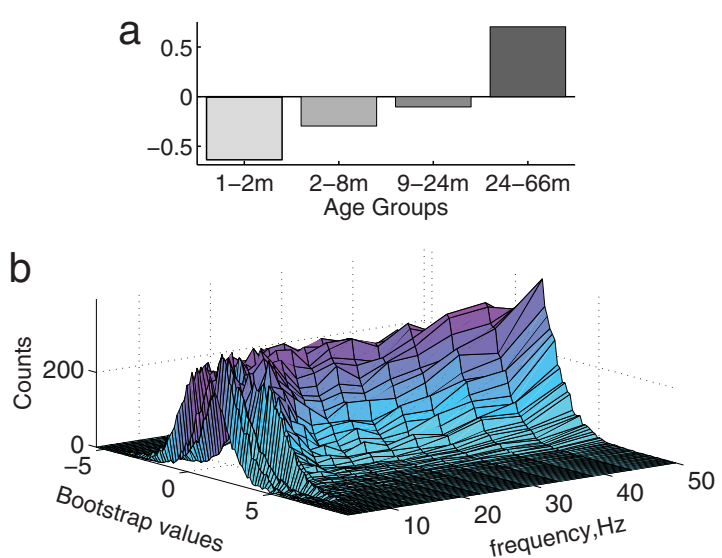

C

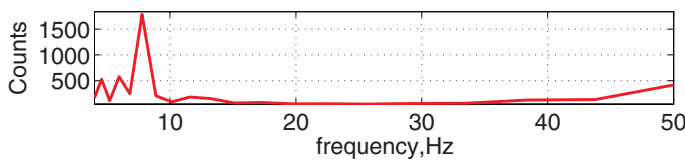

d

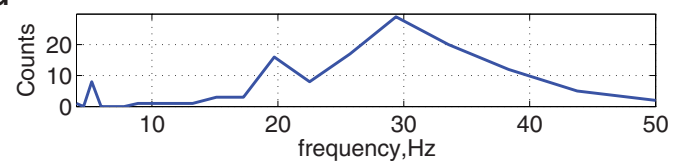

Figure 3. Age-related changes in distributed entropy in auditory condition: underlying contrast from the corresponding PLS analysis $(\boldsymbol{a})$; distribution of the PLS bootstrap ratio values across frequencies $(\boldsymbol{b})$; number of connections with the bootstrap ratio values $>3.5$, which corresponds approximately to the $99 \%$ confidence interval (c); and number of connections with the bootstrap ratio values less than $-3.5(\boldsymbol{d})$. Note the difference in scales on the ordinate axes of $c$ and $\boldsymbol{d}$.
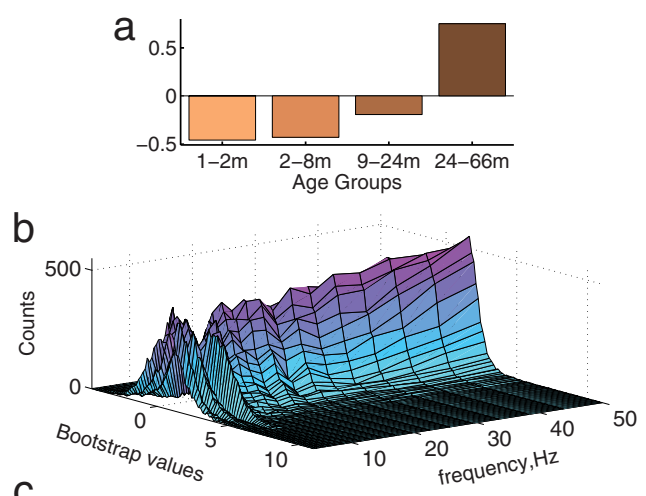

C

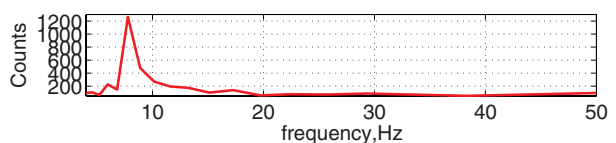

d

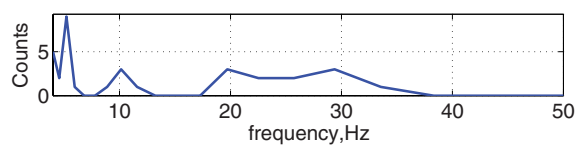

Figure 4. Age-related changes in distributed entropy in visual condition: underlying contrast from the corresponding PLS analysis $(\boldsymbol{a})$; distribution of the PLS bootstrap ratio values across frequencies $(\boldsymbol{b})$; number of connections with the bootstrap ratio values $>3.5$ (c); and number of connections with the bootstrap ratio values less than $-3.5(\boldsymbol{d})$. Note the difference in scales on the ordinate axes of cand $\boldsymbol{d}$.

changes in distributed signal variability. As in the case with local entropy, one significant LV was found both for the auditory and visual task. Figures $3 a$ and $4 a$ illustrate the corresponding datadriven contrasts ( $p<0.001$ in both cases) underlying the group 
differences in terms of mutual information between electrodes in the auditory and visual conditions, respectively. In conjunction with the corresponding distributions of the bootstrap ratio values, the identified contrasts are, on average, interpreted as an increase in the amounts of distributed signal variability with brain development.

The distributions of the bootstrap ratio values that are associated with the contribution of all the electrode pairs to the LV contrast are shown in Figures $3 b$ and $4 b$ as functions of frequency. Specifically, the bootstrap ratios are limited approximately between -5 and 12 . At all the frequencies, the bootstrap ratio distribution is skewed toward positive values, which directly supports the contrasts in Figures $3 a$ and $4 a$.

To be more specific, the effect associated with the developmental changes in connectivity is the most strongly expressed around $8 \mathrm{~Hz}$. Furthermore, the number of connections with the bootstrap ratio values exceeding the threshold of 3.5 is shown in Figures 3, $c$ and $d$, and 4, $c$ and $d$. Relatively large number of connections with high positive bootstrap values can be observed at lower frequencies, with a sharp peak around $8 \mathrm{~Hz}$ for both the auditory and visual tasks. The number of connections that robustly contribute (with 99\% confidence) to the identified contrast is negligibly small compared to the connections supporting the reverse (negative) interpretation of the contrast.

Figure 5 shows spatial distributions of the connections supporting the developmental changes in distributed entropy in the auditory (Fig. 5a,b) and visual (Fig. $5 c, d$ ) conditions. Specifically, Figure 5, $a$ and $c$, shows the connections that directly support the identified contrasts (Figs. $3 a$ and $4 a$, respectively) at $8 \mathrm{~Hz}$ with the bootstrap ratio values $>6$. The distributed entropy for such connections increases with age. The reversed support of the same contrast is shown in Figure $5, b$ and $d$, whereas the blue lines represent connections with the bootstrap ratio values less than -3 at $23-33 \mathrm{~Hz}$. These connections are characterized by a decreased amount of the distributed entropy with development. Because the direct support in the alpha range is a much stronger effect compared to the inverse support in the higher beta to lower gamma range, for the purpose of visualizing the peaks in the topographic maps, we used the different thresholds for the bootstrap ratio values.

\section{Phase locking}

Phase locking in the electrode space was computed as described in Materials and Methods, Phase locking. Similar to Figures 3 and 4, Figures 6 and 7 illustrate the patterns characterizing developmental changes in the phase locking between the EEG channels. PLS mean-centered statistical analyses detected a pattern of linear increase in the amount of phase synchronization (Figs. $6 a, 7 a$ ), which was significant both for the auditory and visual conditions with the $p$ values $<0.001$.
Auditory b
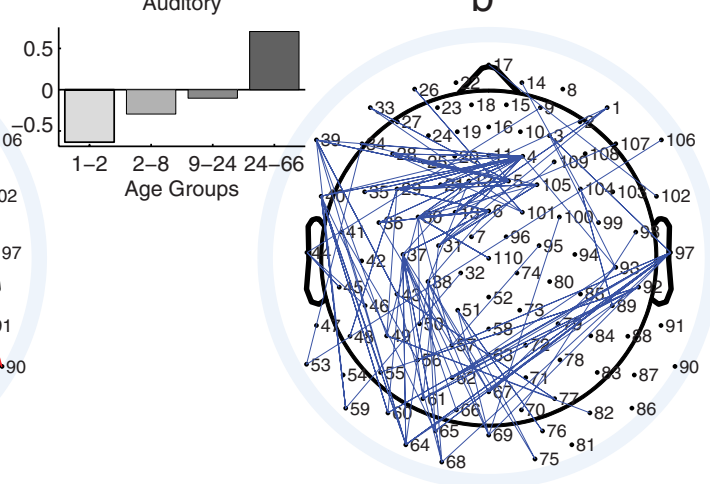

Visual d
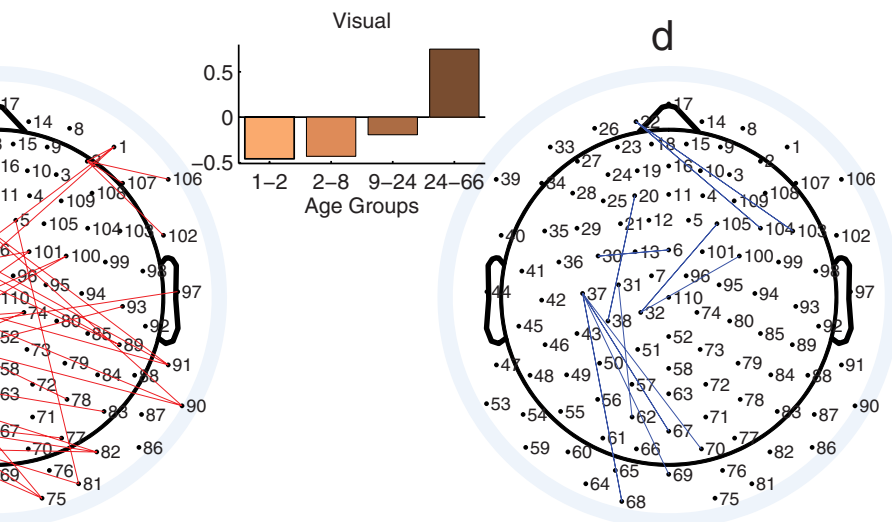
2-8 $9-2424-66$
Age Groups

Figure 5. Spatial distributions of the connections supporting the developmental changes in distributed entropy: in auditory condition, as identified in Figure $3 a(\boldsymbol{a}, \boldsymbol{b})$; and in visual condition, as identified in Figure $4 a(\boldsymbol{c}, \boldsymbol{d})$. The maps in $\boldsymbol{a}$ and $\boldsymbol{c}$ show the connections that directly support the identified contrasts at $8 \mathrm{~Hz}$ with the bootstrap ratio values $>6$. The distributed entropy for $\mathrm{Hz}$ (with the bootstrap ratio values less than -3 ). Such connections shown as the blue lines are characterized by a decreased amount of the distributed entropy with development.

Figures $6 b$ and $7 b$ show the distribution of the bootstrap ratio values associated with pairs of electrodes across the frequencies from 0 to $50 \mathrm{~Hz}$. The number of connections with the bootstrap ratio values exceeding the threshold of 3.5 and -3.5 are plotted as a function of the frequency in $c$ and $d$, respectively, of Figures 6 and 7. The strongest effect is robustly expressed at lower frequencies, directly supporting the increase in the degree of phase locking. It is interesting to note the existence of a peak around $8-9 \mathrm{~Hz}$, which corresponds to the same peak for the distributed entropy. At the same time, it is worth noting that the peak in the distribution of the positive bootstrap ratio values for the mutual information is not as sharp as that for the phase locking.

In addition, there is a robust effect, although not so strong, supporting the inverse interpretation of the contrast shown in Figures $6 a$ and $7 a$. Specifically, in the higher beta frequency range, peaking at $28-30 \mathrm{~Hz}$, the degree of phase locking is decreasing with age. Comparing Figures $3 d$ and $6 d$, or Figures $4 d$ and $7 d$, one would note that the analysis based on the mutual information between signal amplitudes hints at the existence of the same pattern of a linear decrease in phase synchronization with age. Still, in terms of the number of connections with the high bootstrap ratio values, this effect is weaker for the distributed entropy than for the phase locking.

Similar to Figure 5, Figure 8 shows spatial distributions of the connections supporting the developmental changes in the degree of phase locking between the electrodes in the auditory (Fig. 8a,b) and visual (Fig. $8 c, d$ ) conditions. Specifically, the connections 

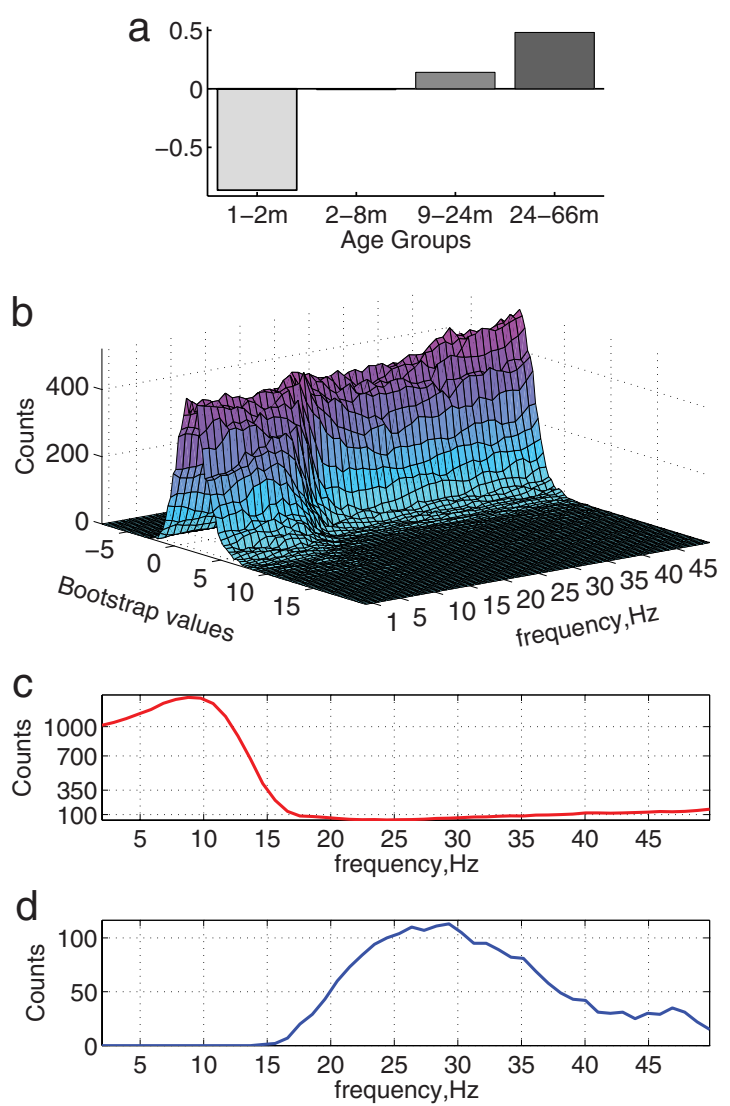

Figure 6. Differences in phase locking across the age groups in auditory condition: pattern characterizing developmental changes $(\boldsymbol{a})$; distribution of the corresponding PLS bootstrap ratio values across frequencies $(\boldsymbol{b})$; number of connections with the bootstrap ratio values $>3.5$ (c); and number of connections with the bootstrap ratio values less than -3.5 (d).

that directly support the identified contrasts (Figs. $6 a, 7 a$ ) at $8-11$ $\mathrm{Hz}$ are shown in Figure 8, $a$ and $c$, as the red lines. The degree of phase locking for such connections increases with age. The blue lines in Figure 8, $b$ and $d$, represent the connections at $23-33 \mathrm{~Hz}$ for which the degree of phase locking decreases in development (the inverse support of the same contrasts). Similar to Figure 5, for better visualizations of the peaks in the topographic maps, the thresholds for the bootstrap ratio values were set at $>6$ and less than -3 for the support in the alpha and higher beta to lower gamma range, respectively.

\section{Discussion}

As the brain develops, its activity becomes more variable and complex. Two key mechanisms may contribute to brain complexity: bigger repertoire of the physiological states of individual regions that become more specialized, and increased integration between distributed neuronal populations. We showed that the latter mechanism is a key factor contributing to the increased complexity of brain signals in development. The degree of integration of distributed neuronal populations in the brain increases with age. These effects can be observed both in terms of nonlinear correlations between signal amplitudes (mutual information) and phase synchronization. Moreover, this is accompanied by a decrease in the amount of information processed locally, quantified as average conditional entropy. In other words, the variability of brain dynamics associated with differentiating spatially local information transforms into the variability of coordinated brain activity during the development.
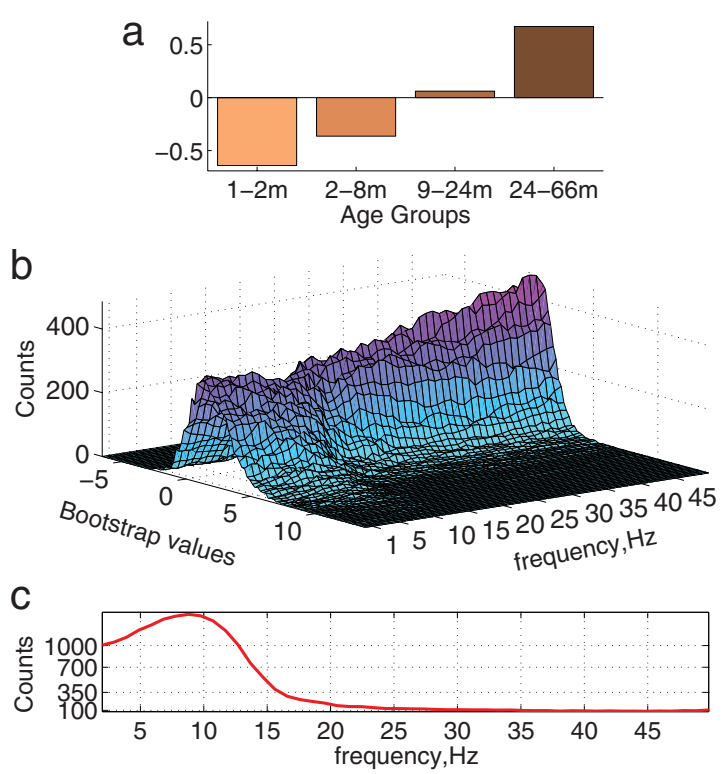

d

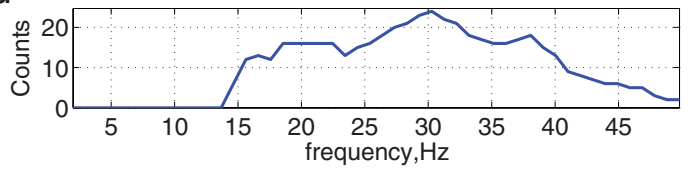

Figure 7. Differences in phase locking across the age groups in visual condition: pattern characterizing developmental changes $(\boldsymbol{a})$; distribution of the corresponding PLS bootstrap ratio values across frequencies $(\boldsymbol{b})$; number of connections with the bootstrap ratio values $>3.5$ (c); number of connections with the bootstrap ratio values less than -3.5 (d).

It should be clarified that specialization of individual brain regions (modules of the underlying functional networks) may increase with development. This is in line with other evidence indicating increased refinement of neuronal maps and receptive fields. Under the framework of metastability, this could be interpreted as a greater repertoire of the microstates of individual modules and more facile state transitions (Fingelkurts and Fingelkurts, 2004). In turn, the increased number of microstates can be observed though the corresponding increased signal complexity, which has been shown in a number of studies (MeyerLindenberg, 1996; McIntosh et al., 2008). It is worth noting that estimating signal complexity essentially quantifies the variability of the brain signals, and thus can be associated with the total entropy of individual modules constituting a network. In this study, we attempted to decompose this total amount of entropy. Specifically, the local entropy defined by conditioning over distant modules was shown to be decreasing in development. However, this does not contradict the fact that local specialization increases in parallel, which is reflected by the increased total entropy. Such a phenomenon is possible as entropy assigned to specific modules becomes more and more efficiently shared.

Increased connectivity was observed at lower frequencies, reaching a peak around $8-10 \mathrm{~Hz}$. At the same time, these effects were accompanied by increased phase scattering at higher frequencies, in the higher beta or lower gamma range. The analyses based on mutual information indicated the presence of such effects in terms of correlations between signal amplitudes. Still, the desynchronization effect at higher frequencies, although being weaker than increased synchrony, was clearly observed in terms of phase locking over pairs of the electrodes. Such findings would support the hypothesis that the interplay between phase locking and phase desynchronization across different frequency bands is 
a crucial factor for the process of largescale integration (Varela et al., 2001). The possible mechanism involves inhibitory modulations of the neural rhythms, leading to phase resetting of underlying neural ensembles. In other words, uncoupling of the connected neural populations, observed as active phase scattering rather than a return to the baseline of synchrony, is necessary to switch from one cognitive state to another (Rodriguez et al., 1999). In turn, a greater repertoire of dynamic states in development would imply an increased demand for the computational resources related to active phase desynchronization.

Computational modeling demonstrated that phase scattering marking the transition between synchronized states is an efficient and flexible method of integration of dynamic systems. This was done under the framework of viewing the brain as an ensemble of coupled nonlinear systems (Nunez, 1995). Specifically, a number of studies reported the presence of desynchronization patterns from chaotic states in coupled oscillator systems. The arrays of oscillators can be synchronized with weak coupling. However, either the coupling strength or the number of coupled systems exceeding a critical threshold can lead to the appearance of desynchronizing bifurcations. Physiologically, it may reflect the myelination of cortical pathways (Paus et al., 1999), changes in synaptic density (Huttenlocher and Dabholkar, 1997), and brain volume (Courchesne et al., 2000).

It would be interesting to look into the transformation of local signal variability into connectivity in development from the perspective of studies dedicated to consciousness. Regarding this long-standing mystery, a key issue is to find mechanisms responsible for our being aware of some thoughts and feelings. A number of studies have emphasized the integrative function of consciousness (Tononi and Edelman, 1998). Global workspace theory involves a notion of fleeting memory and works under the hypothesis that only one consistent event corresponding to what we are conscious of can be dominant at a given moment (Baars, 2002, 2005). This dominant content should be widely distributed across the brain. One of the predictions of this model is related to an interplay between widespread and local information. Specifically, according to the model, nonconscious experiences are processed locally within separate brain areas. At the same time, conscious perception arises when the dominant events are broadcast to a wider network of unconscious brain processes. The result is the appearance of long-range coordinated activity, corresponding to the widespread flows of information across the global workspace. Our findings regarding the transformation of local entropy into connectivity in the alpha range seem to be in good accordance with a theory claiming that consciousness is integrated information (Tononi, 2004, 2008). At the same time, disintegration observed at higher frequencies can also be viewed under the same framework. Broadcasting local information to a network of neural regions should require the ability of the brain found to be decreasing with age. a

b
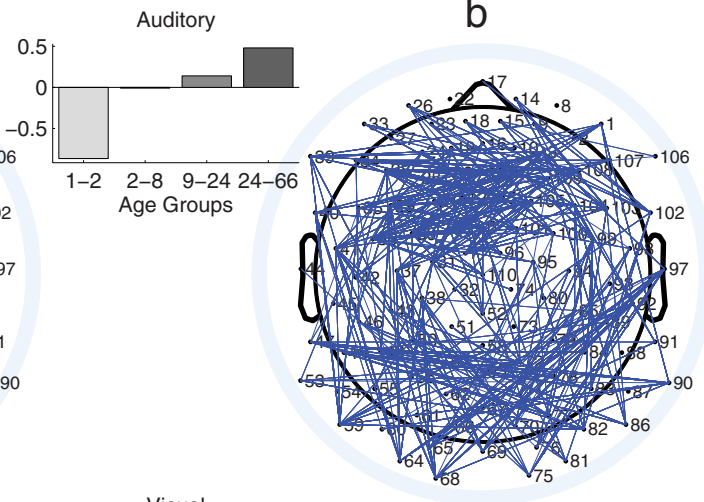

$d$

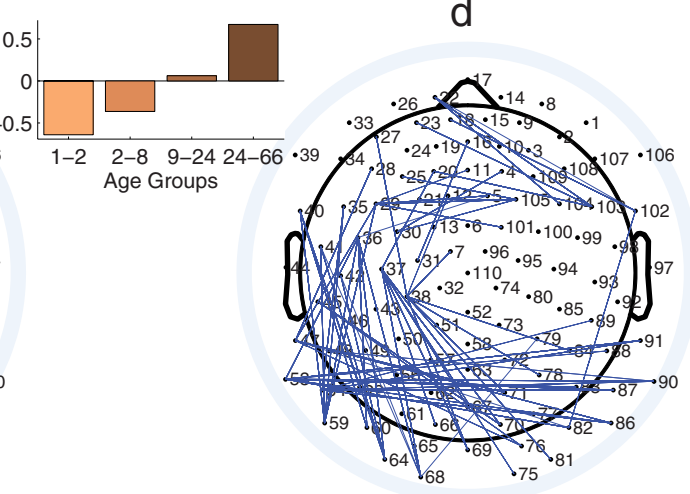

Figure 8. Spatial distributions of the connections supporting the developmental changes in phase locking: in auditory condisame contrasts at $23-33 \mathrm{~Hz}$ (with the bootstrap ratio values less than -3 ). The degree of phase locking for such connections was

to deal with neural conflicts. The mechanism to prevent conflicting messages from being broadcast to the global workspace could be based on inhibition destroying synchronized processes.

Despite the fact that global workspace theory seems to be consistent with our findings at first approximation, we should clarify the question about differentiating between high integration in a network and high information transferred through this network. As implied by global broadcasting of signals from any particular cortical module, the same message or conscious content would be sent to many modules constituting a network. Such a situation requires that the coupling (integration) between different modules be relatively high. However, this does not exclude the idea that a single strong signal being broadcast to the rest of the brain represents high integration but little information transfer. The observed increase in mutual information between distant modules is more compatible with the idea that different signals, as carriers of information, are being exchanged effectively among many different modules. In other words, each module can strongly affect many targets, and different modules do so in different ways, which represents the simultaneous existence of both high integration and high information exchange. It is interesting to note that the high information being transferred requires both synchronization and desynchronization at different frequency bands, as suggested by computer simulations of neuron models. Specifically, as shown in Baptista and Kurths (2008), the maximum capacity of information transfer between neurons can be reached if the neurons synchronize at 
slow timescales (lower frequencies) and desynchronize at fast timescales (higher frequencies).

\section{References}

Baars BJ (2002) The conscious access hypothesis: origins and recent evidence. Trends Cogn Sci 6:47-52.

Baars BJ (2005) Global workspace theory of consciousness: toward a cognitive neuroscience of human experience. Prog Brain Res 150:45-53.

Baddeley R, Hancock P, P Földiák, eds (2000) Information theory and the brain. Cambridge, UK: Cambridge UP.

Baptista MS, Kurths J (2008) Transmission of information in active networks. Phys Rev E Stat Nonlin Soft Matter Phys 77:026205.

Chávez M, Le Van Quyen M, Navarro V, Baulac M, Martinerie J (2003) Spatio-temporal dynamics prior to neocortical seizures: amplitude versus phase couplings. IEEE Trans Biomed Eng 50:571-583.

Churchland PS, Sejnowski TJ (1988) Perspectives on cognitive neuroscience. Science 242:741-745.

Cohen JD, Tong F (2001) Neuroscience: the face of controversy. Science 293:2405-2407.

Costa M, Goldberger AL, Peng CK (2002) Multiscale entropy analysis of physiologic time series. Phys Rev Lett 89:068102.

Courchesne E, Chisum HJ, Townsend J, Cowles A, Covington J, Egaas B, Harwood M, Hinds S, Press GA (2000) Normal brain development and aging: quantitative analysis at in vivo MR imaging in healthy volunteers. Radiology 216:672-682.

Deisboeck TS, Kresh JY, eds (2006) Complex systems in science in biomedicine. New York: Springer.

Delorme A, Makeig S (2004) EEGLAB: an open source toolbox for analysis of single-trial EEG dynamics including independent component analysis. J Neurosci Methods 134:9-21.

Düzel E, Habib R, Schott B, Schoenfeld A, Lobaugh N, McIntosh AR, Scholz M, Heinze HJ (2003) A multivariate, spatiotemporal analysis of electromagnetic time-frequency data of recognition memory. Neuroimage 18:185-197.

Fingelkurts AA, Fingelkurts AA (2004) Making complexity simpler: multivariability and metastability in the brain. Int J Neurosci 114:843-862.

Friston KJ (1998) Imaging neuroscience: principles or maps? Proc Natl Acad Sci U S A 95:796-802.

Hay JF, Kane KA, West R, Alain C (2002) Event-related neural activity associated with habit and recollection. Neuropsychologia 40:260-270.

Huttenlocher PR, Dabholkar AS (1997) Regional differences in synaptogenesis in human cerebral cortex. J Comp Neurol 387:167-178.

Jirsa VK, McIntosh AR, eds (2007) Handbook of brain connectivity. Berlin: Springer.

Lippé S, Kovacevic N, McIntosh AR (2009) Differential maturation of brain signal complexity in the human auditory and visual system. Front Hum Neurosci 3:48.

Lippé S, Roy M-S, Perchet C, Lassonde M (2007) Electrophysiological markers of visuocortical development. Cereb Cortex 17:100-107.

Lobaugh NJ, West R, McIntosh AR (2001) Spatiotemporal analysis of ex- perimental differences in event-related potential data with partial least squares. Psychophysiology 38:517-530.

McIntosh AR, Lobaugh NJ (2004) Partial least squares analysis of neuroimaging data: applications and advances. Neuroimage 23:S250-S263.

McIntosh AR, Kovacevic N, Itier RJ (2008) Increased brain signal variability accompanies lower behavioral variability in development. PLoS Comput Biol 4:e1000106.

Meyer-Lindenberg A (1996) The evolution of complexity in human brain development: an EEG study. Electroencephalogr Clin Neurophysiol 99:405-411.

Mormann F, Lehnertz K, David P, Elger EC (2000) Mean phase coherence as a measure for phase synchronization and its application to the EEG of epilepsy patients. Physica D 144:358-369.

Nunez PL (1995) Neocortical dynamics and human EEG rhythms. Oxford: Oxford UP.

Paus T, Zijdenbos A, Worsley K, Collins DL, Blumenthal J, Giedd JN, Rapoport JL, Evans AC (1999) Structural maturation of neural pathways in children and adolescents: in vivo study. Science 283:1908-1911.

Prichard D, Theiler J (1995) Generalized redundancies for time series analysis. Physica D 84:476-493.

Rodriguez E, George N, Lachaux JP, Martinerie J, Renault B, Varela FJ (1999) Perception's shadow: long-distance synchronization of human brain activity. Nature 397:430-433.

Rosenblum MG, Pikovsky AS, Kurths J (1996) Phase synchronization of chaotic oscillators. Phys Rev Lett 76:1804-1807.

Shannon CE (1949) The mathematical theory of communication. Urbana, IL: University of Illinois.

Silverman BW (1986) Density estimation for statistics and data analysis. London: Chapman Hall.

Small M, Tse CK (2004) Optimal embedding parameters: a modeling paradigm. Physica D 194:283-296.

Stam CJ (2005) Nonlinear dynamical analysis of EEG and MEG: review of an emerging field. Clin Neurophysiol 116:2266-2301.

Stam CJ, Nolte G, Daffertshofer A (2007) Phase lag index: assessment of functional connectivity from multi channel EEG and MEG with diminished bias from common sources. Hum Brain Mapp 28:1178-1193.

Tass P, Rosenblum MG, Weule J, Kurths J, Pikovsky A, Volkmann J, Schnitzler A, Freund H-J (1998) Detection of n:m phase locking from noisy data: application to magnetoencephalography. Phys Rev Lett 81:3291-3294.

Tononi G (2004) An information integration theory of consciousness. BMC Neurosci 5:42.

Tononi G (2008) Consciousness as integrated information: a provisional manifesto. Biol Bull 215:216-242.

Tononi G, Edelman GM (1998) Consciousness and complexity. Science 282:1846-1851.

Tononi G, Sporns O, Edelman GM (1994) A measure for brain complexity: relating functional segregation and integration in the nervous system. Proc Natl Acad Sci U S A 91:5033-5037.

Varela F, Lachaux JP, Rodriguez E, Martinerie J (2001) The brainweb: phase synchronization and large-scale integration. Nat Rev Neurosci 2:229239. 